\title{
Fungos da Família Pucciniaceae Causadores de Ferrugem em Plantas Medicinais*
}

\author{
Cristiano S. Lima**, Paulo E. de Souza \& Alex O. Botelho** \\ Departamento de Fitopatologia, Universidade Federal de Lavras, Cx. Postal 37, CEP 37200-000, Lavras, MG, \\ e-mail: cslima78@yahoo.com.br
}

(Aceito para publicação em 19/05/2004)

Autor para correspondência: Cristiano S. Lima

LIMA, C.S., SOUZA, P.E. de \& BOTELHO, A.O. Fungos da família Pucciniaceae causadores de ferrugem em plantas medicinais. Fitopatologia Brasileira 29:499-503. 2004.

\section{RESUMO}

Por meio do estudo de estruturas fúngicas ao microscópio estereoscópico e ótico e de uma caracterização em detalhes, inclusive com micrografias, foi possível identificar cinco espécies de fungos causadores de ferrugens da família Pucciniaceae associadas com sete espécies vegetais medicinais do horto Ervas \& Matos da Universidade Federal de Lavras, em Minas Gerais (21⒈ $14^{\prime}$ - latitude sul; $45^{\circ} 00^{\prime}$ - longitude oeste). As espécies de ferrugem e seus respectivos hospedeiros foram: Puccinia lantanae em Lippia alba; P. leonotidicola em Leonotis nepetaefolia; P. menthae em Mentha spp. e M. arvensis; P. porophylli em Porophyllum ruderale; e Uromyces platensis sobre Pfaffia glomerata

Palavras-chave adicionais: Uredinales, identificação, doença.

\begin{abstract}
Rust fungi of the Pucciniaceae family on medicinal plants

This paper brings a detailed characterization including the geographical distribution of four Puccinia and one Uromyces species. According to the results Puccinia lantanae was found on

Lippia alba; P. leonotidicola on Leonotis nepetaefolia; P. menthae on Mentha spp. and Mentha arvensis; P. porophylli on Porophyllum ruderale and Uromyces platensis on Pfaffia glomerata.
\end{abstract}

\section{INTRODUÇÃO}

As plantas medicinais são conceituadas como vegetais capazes de elaborar substâncias com ação farmacológica, benéfica ou prejudicial sobre o organismo humano (Martins et al., 1995). Estas têm importância reconhecida como fitoterápicos simples e como matéria-prima para a indústria farmacêutica (Mattos, 1983; Karl et al., 1997).

A família de fungos Pucciniaceae encontra-se dentro da ordem Uredinales, classe Teliomycetes e filo Basidiomycota, apresentando os gêneros Puccinia e Uromyces como os mais importantes em termos agrícolas e econômicos, os quais possuem patógenos obrigatórios em diversas plantas cultivadas e selvagens em praticamente todos os continentes, causando sérias perdas em plantios comerciais por interferir com a fisiologia do hospedeiro, reduzindo a produtividade e depreciando os produtos agrícolas (Cummins \& Hiratsuka, 1983; Hawksworth et al., 1995, Agrios, 1997).

O gênero Puccinia possui em torno de 4.000 espécies em vários hospedeiros (Hawksworth et al., 1995). A espécie tipo é Puccinia graminis Pers. ex Pers. (Cummins \& Hiratsuka, 1983). O gênero Uromyces possui 600 espécies as quais estão amplamente distribuídas em termos geográficos e de

\footnotetext{
*Parte da dissertação de mestrado do primeiro autor. Departamento de Fitopatologia. Universidade Federal de Lavras. (2002)

**Bolsista CNPq
}

hospedeiros (Hawksworth et al., 1995). A espécie tipo é Uromyces appendiculatus (Pers.) Unger (Cummins \& Hiratsuka, 1983).

Conhecer as doenças das plantas medicinais é importante quando se pensa na adoção de medidas de manejo integrado e no aumento da produtividade. Porém, poucos trabalhos têm sido publicados no Brasil sobre as relações entre plantas medicinais e fitopatógenos (Urben et al., 1987; Souza et al., 1995).

A correta identificação do patógeno consiste no primeiro passo em direção ao posterior manejo da doença por ele causada. Este trabalho teve como objetivo acrescentar informações sobre espécies causadoras de ferrugem da família Pucciniaceae em plantas medicinais encontradas no horto Ervas \& Matos da Universidade Federal de Lavras, incluindo também sintomatologia e distribuição geográfica.

\section{MATERIALEMÉTODOS}

Os materiais vegetais infetados foram coletados, fotografados, exsicatados e depositados no Herbário Micológico de Lavras (HML) mantido pelo Laboratório de Sistemática e Ecologia de Fungos da Universidade Federal de Lavras (UFLA). Preparações microscópicas em ácido láctico contendo estruturas e esporos dos patógenos foram também depositadas. Todo o material vegetal foi registrado na Clínica 


\section{C.S. Lima et al.}

Fitossanitária do Departamento de Fitopatologia da UFLA.

Uma parte do material foi separada para as observações de sintomas e sinais dos patógenos utilizando microscópio estereoscópico e ótico. Para a preparação microscópica, foram utilizadas lâminas e lamínulas de vidro, estiletes para a raspagem das estruturas fúngicas e lâminas de barbear para a preparação de cortes manuais sob microscópio estereoscópico. Ácido láctico foi utilizado como líquido de montagem e as lâminas foram seladas com esmalte de unhas. As medições das estruturas fúngicas e esporos de cada espécie foram realizadas em microscópio ótico, obtendo-se a média de 30 medições para cada estrutura.

\section{RESULTADOS E DISCUSSÃO}

A seguir serão apresentadas e discutidas as espécies da família Pucciniaeceae encontradas em plantas medicinais do horto Ervas \& Matos localizado no campus da Universidade Federal de Lavras, MG.

Puccinia lantanae Farlow, Proc. Amer. Acad. 18: 83. 1883. Sinonímia: Uromyces lantanae Spegazzini, Anal. Soc. Cient. Argentina 17:93. 1884; Puccinia elytrariae P. Hennings, Hedwigia 34:320. 1895; P. accedens P. \& H. Sydow, Mon. Ured. 1:309.1902.

Urédios e Télios (0,3 a 2,5 mm de diâmetro) mistos, hipófilos e de coloração parda. Lesões com halos amarelados nítidos. Urediniósporos (19-27 x 17-22 $\mu \mathrm{m})$, numerosos, piriformes ou globosos, unicelulares, pardo-castanhos, lisos, pedicelados, em grande quantidade (Figura 1). Teliósporos (2232 x 15-19,5 $\mu \mathrm{m}$ ), elipsóides na maioria, bicelulares, pardocastanhos, produzidos em pequena quantidade, lisos, constritos no septo, base rotundada, pedicelo persistente, fusco, dilatado junto à inserção, por vezes inserindo-se obliquamente, pedicelo às vezes septado (Figura 1 ).

Hospedeiro: Erva cidreira-de-arbusto [Lippia alba (Mill) N.E. Brown], planta medicinal da família Verbenaceae, originária da América do Sul. O seu preparado tem efeito calmante, atua contra insônia e possui efeito estomáquico.

Material examinado: HML 144, Lavras, MG, 23 de julho de 2001.

Este fungo foi identificado como sendo P. lantanae, em concordância com o relato dessa espécie feito por Viégas (1945).

Esta espécie de ferrugem foi relatada em Lippia spp. em Cuba, no México e na Venezuela (Farr et al., 2003), constatada no Brasil em Lippia aristata Schauer no estado do Mato Grosso, Lippia rhodocnemis Martens \& Schaurer no estado do Rio de Janeiro, em Lippia sp. em São Paulo (Hennen et al., 1982) e em L. alba no estado do Paraná (Schuta et al., 1997).

Puccinia leonotidicola P. Hennings, In: H. Baum, Bot. Ergebnisse der Kunene-Sambesi Exped., p. 2, 1903.

Sinonímia:Uredo leonotidis P. Hennings, In: Engler, Pflanz. Ost. Afr. Teil C, 3:52. 1895; U. leonotidicola P. Hennings,
Hedwigia Beiblatt 38:(69). 1899; Puccinia leonotidis (P. Hennings) Arthur, Mycologia 7:245. 1915; P. dominicana Gonzales-Fragoso \& Cifferri, Bol. R. Soc. Espan. Hist. Nat. 26:248.1926.

Télios não vistos. Urédios ( 0,5 a 2,0 mm de diâmetro), castanhos, anfígeno (Figura 2A). Lesões com halos amarelados, coalescentes. Urediniósporos (19,5 - 27 x 18,5 - 23 $\mu \mathrm{m})$, equinulados, globosos, deprimidos, pardo-avermelhados, paredes com 1 a $1,5 \mu \mathrm{m}$ de espessura, três a cinco poros germinativos sub-equatoriais (Figura 2B).

Hospedeiro: Cordão-de-frade [Leonotis nepetaefolia (L.) R. Br.], planta herbácea da família Labiatae, tendo origem na África tropical e meridional e Índias orientais. É empregada como tônico e balsâmico das vias respiratórias e urinárias.

Material examinado: HML 136, Lavras, MG, 18 de junho de 2001.

Este fungo foi identificado como sendo $P$. leonotidicola, de acordo com o relato feito por Viégas (1945).

Existem relatos da espécie em Cuba, Madagascar, México, Porto Rico, Ilhas Virgens, Colômbia, Trinidade e Tobago (Farr et al., 2003) e no Brasil (Mendes et al., 1998). No Brasil, $P$. leonotidicola foi relatada nos estados de Minas Gerais, Pernambuco, São Paulo, Santa Catarina, Rio Grande do Sul e Rio de Janeiro (Hennen et al., 1982; Urben et al., 1987; Mendes et al., 1998). Puccinia sp. relatada em cordão-de-frade no Distrito Federal, possivelmente, deve-se tratar de $P$. leonotidicola, pois, de acordo com Viégas (1945), esta espécie de fungo causador de ferrugem é comum em L. nepetaefolia no Brasil. Contrastando com o relato de Viégas (1945), o qual faz referência a três-quatro poros germinativos, foram observados urediniósporos com até cinco poros germinativos no material aqui estudado.

Puccinia menthae Persoon, Syn. Meth. Fung., 227. 1801. Espermogônios, écios e télios não vistos. Urédios (0,5 a $1 \mathrm{~mm}$ de diâmetro), coloração castanho-claro. Halos cloróticos na parte adaxial da folha ao longo do limbo foliar, que mais tarde surgem na face abaxial (Figura 3A). Urediniósporos (1727 x 15-24,5 $\mu \mathrm{m}$ ), globosos a elípticos, equinulados, paredes variando de 1,5 a $2,5 \mu \mathrm{m}$, amarelados, com dois a três poros equatoriais (Figura 3B).

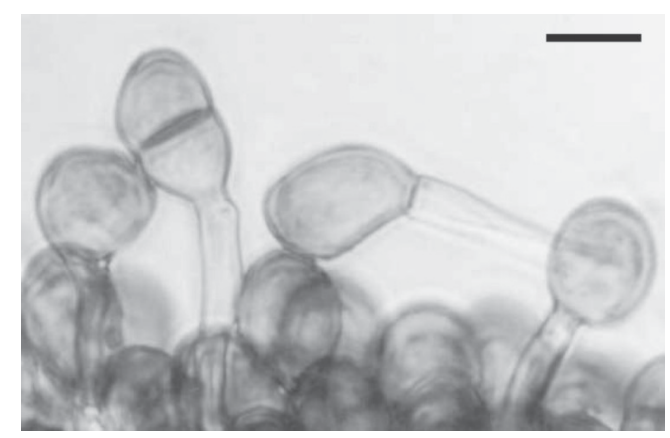

FIG. 1 - Puccinia lantanae sobre Lippia alba. Teliósporo bicelular visto ao microscópio ótico (MO) (lado esquerdo). Urediniósporo unicelular visto ao MO (lado direito). Barra: $20 \mu \mathrm{m}$. 


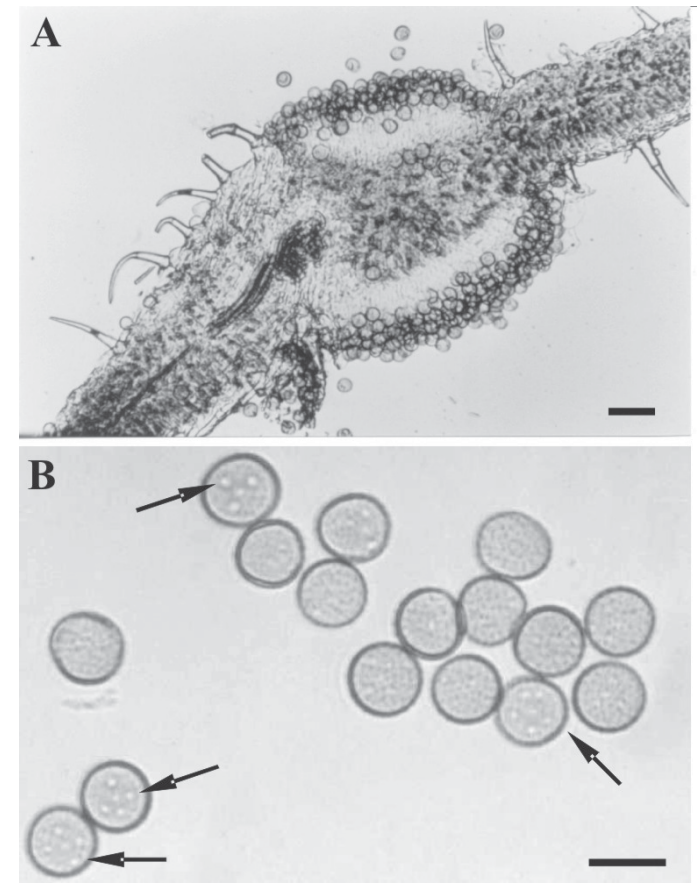

FIG. 2 - A - B. Puccinia leonotidicola sobre Leonotis nepetaefolia. A - Corte apresentando urédios anfígenos vistos ao MO. B Urediniósporos vistos ao $\mathrm{MO}$ com poros sub-equatoriais ao redor do ponto de inserção do pedicelo (setas). Barras: $\mathrm{A}=60 \mu \mathrm{m}$; $\mathrm{B}=$ $20 \mu \mathrm{m}$.

Hospedeiros: Hortelã (Mentha sp.), hortelã japonesa (Mentha arvensis L.) e menta (Mentha sp.), são ervas medicinais de porte herbáceo, pertencentes à família Labiatae, de origem na Europa ou vindas do Oriente. Estas plantas são utilizadas como descongestionante nasal, antiemético, vermífugo, carminativo e no combate à cefaléia.

Material examinado: Hortelã - HML 140, Lavras, MG, 25 de junho de 2001; Hortelã Japonesa - HML 139, Lavras, MG, 25 de junho de 2001; Menta - HML 138, Lavras, MG, 25 de junho de 2001.

Pelo estudo das características morfológicas do fungo foi possível identificá-lo como $P$. menthae.

Esta espécie de ferrugem é relatada em praticamente todo o mundo em uma variada gama de espécies do gênero Mentha. Trata-se da principal espécie de ferrugem relacionada a esse gênero (Farr et al., 2003). Possui duas variedades: $P$. menthae var. menthae e $P$. menthae var. pseudomenthae.

$O$ fungo estudado traz grandes semelhanças morfológicas com o relato feito por Viégas (1945), tendo o mesmo causado sérios danos a plantios de Mentha spp. no estado de São Paulo. No Brasil, existem relatos desta espécie de ferrugem em $M$. arvensis e $M$. piperita em São Paulo e Mentha sp. no Paraná e em São Paulo (Hennen et al., 1982; Mendes et al., 1998). Em um levantamento de doenças em plantas medicinais realizado no Distrito Federal registrou-se Puccinia sp. para M. spicata L. e Mentha sp., sendo P. menthae relatada para $M$. arvensis e M. piperita L. (Urben et al., 1987).
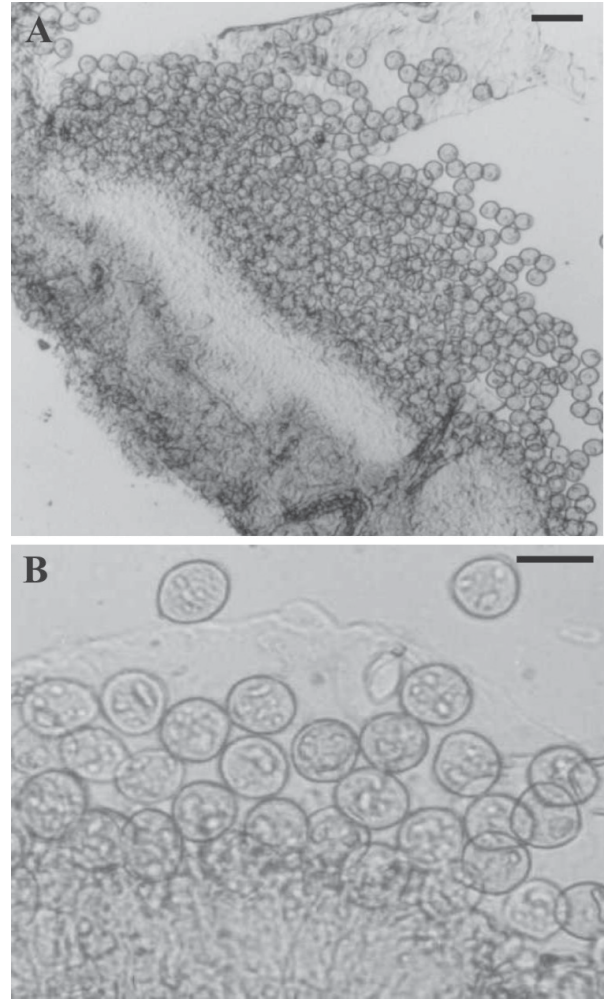

FIG. 3 - A - B. Puccinia menthae sobre Mentha spp. A - Corte apresentando urédios vistos ao MO. B - Urediniósporos vistos ao MO. Barras: $\mathrm{A}=60 \mu \mathrm{m} ; \mathrm{B}=20 \mu \mathrm{m}$.

Puccinia porophylli P. Hennings, Hedwigia Beiblatt 39:(153). 1900.

Sinonímia: Uredo aperta Winter, In: Rabenhorst, Hedwigia 26:24.1887.

Télios não vistos. Urédios (0,5 a 1 mm de diâmetro), anfígenos, coloração pardo-castanho, distribuídos ao longo do limbo foliar. Urediniósporos (18,5-27 x 17-24,5 $\mu \mathrm{m})$, globosos a elípticos, de paredes finamente equinuladas, 2 a $2,5 \mu \mathrm{m}$ de espessura (Figura 4).

Hospedeiro: Arnica paulista [Porophyllum ruderale (Jacq.) Cass.], planta herbácea da família Asteraceae, oriunda do Brasil, empregada no tratamento de contusões e dores musculares.

Material examinado: HML 148, Lavras, MG 27 de agosto de 2001.

Pelas observação das estruturas do patógeno constatou-se que a espécie em estudo é $P$. porophylli.

Puccinia porophylli já foi relatada no Brasil, nos estados do Rio Grande do Sul, Santa Catarina, São Paulo e no Distrito Federal (Viégas, 1945; Mendes et al., 1998). Segundo Viégas (1945), esta espécie ocorre desde a América Central até a América do Sul.

Uromyces platensis Spegazzini, An. Soc. Cient. Argent. 12: 27.1881.

Urédios ( 0,8 a 3,0 mm de diâmetro), coloração castanha, anfígenos, principalmente hipófilos, bem distribuídos ao longo 


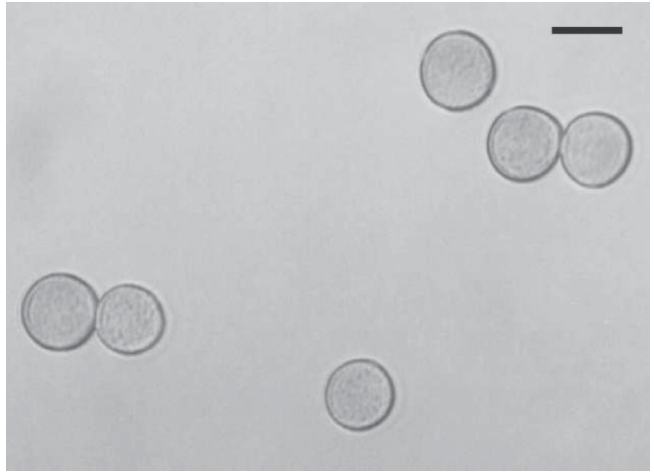

FIG. 4 - Puccinia porophylli sobre Porophyllum ruderale. Urediniósporos vistos ao MO. Barra $=25 \mu \mathrm{m}$

da superfície da folha coalescendo mais tarde, pústulas com halos amarelados discretos ocorrendo manchas esbranquiçadas no verso da folha. Urediniósporos (22-32 x 19-23 $\mu \mathrm{m})$, numerosos, globosos ou obovóides a elípticos, unicelulares, com dois poros germinativos equatoriais, pardo-castanhos, verrugosos, paredes com 2 a $3 \mu \mathrm{m}$ de espessura (Figura 5A). Télios presentes nos ramos do hospedeiro, coloração parda. Teliósporos (34-53 x 25-40 $\mu \mathrm{m})$, elípticos a globosos, unicelulares, pardo-castanhos, lisos, paredes com 2 a $3 \mu \mathrm{m}$ de espessura nas laterais, papila com 6-11 $\mu \mathrm{m}$, pedicelo persistente, hialino (Figura 5B).

Hospedeiro: Acônito [Pfaffia glomerata (A. Spreng.) Pedersen], planta herbácea da família Amaranthaceae. Espécie nativa da América do Sul, possivelmente da Bolívia, Brasil ou Paraguai. Esta planta é utilizada como tônico, antidiabético, antidiarréico e antihemorrágico.

Material examinado: HML 147, Lavras, MG, 27 de agosto de 2001.

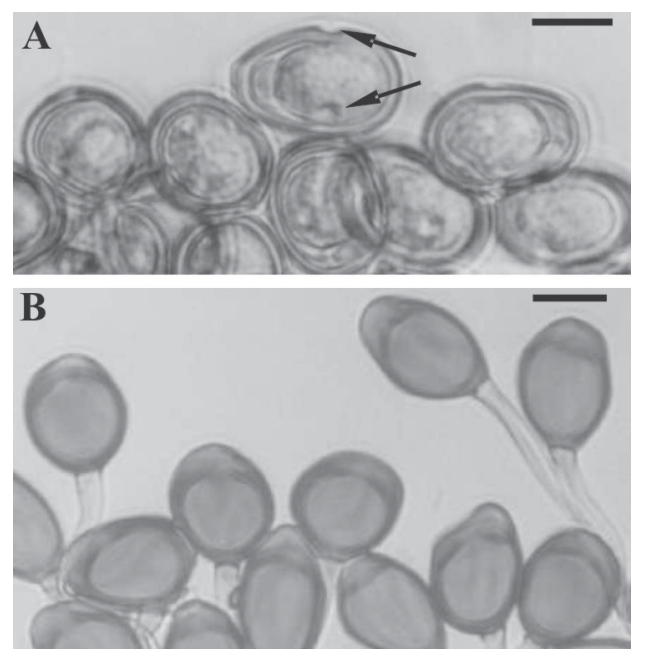

FIG. 5 - A - B. Uromyces platensis sobre Pfaffia glomerata. A Urediniósporos vistos ao MO mostrando poros germinativos equatoriais (setas). B - Teliósporos unicelulares típicos vistos ao MO. Barras: $\mathrm{A}=15 \mu \mathrm{m} ; \mathrm{B}=25 \mu \mathrm{m}$.
Pelas observações das características morfológicas das estruturas do fungo e posterior comparação com a descrição presente na literatura foi possível identificá-lo como sendo $U$. platensis.

Uromyces platensis foi relatado em P. stenophylla na Argentina (Farr et al., 2003). No Brasil, U. platensis é relatada para P. glomerata no Distrito Federal (Mattos \& Dianese, 1992, 1996). Uromyces platensis ocorre também no estado do Mato Grosso do Sul em espécies de Pfaffia (Arias et al., 2001).

\section{AGRADECIMENTOS}

Ao Conselho Nacional de Desenvolvimento Científico e Tecnológico (CNPq) pelo financiamento da bolsa de estudos durante a realização do curso de mestrado de Cristiano Souza Lima e pela bolsa de iniciação científica (programa de bolsas de iniciação científica - PIBIC) de Alex Oliveira Botelho.

\section{REFERÊNCIAS BIBLIOGRÁFICAS}

AGRIOS, N.G. Plant pathology. 4. ed. San Diego: Academic Press, 1997. 635p.

ARIAS, S.M.S., URBEN, A.F. \& LAURA, V.A. Uromyces platensis em Pfaffia spp. no Mato Grosso do Sul. Resumos, $34^{\circ}$ Congresso Brasileiro de Fitopatologia, São Pedro, SP. 2001. p. 359.

CUMMINS, G.B. \& HIRATSUKA, Y. Illustrated Genera of Rust Fungi. 2. ed. rev. Saint Paul:American Phytopathological Society, 1983.

FARR, D.F., ROSSMAN, A.Y., PALM, M.E. \& McCRAY, E.B. (n.d.) Fungal Databases, Systematic Botany \& Mycology Laboratory, ARS, USDA. Disponível em:<http://nt.ars-grin.gov/fungaldatabases/ $>$. Acessos em junho de 2003.

HAWKSWORTH, D.L., KIRK, P.M., SUTTON, B.C. \& PEGLER, D.N. Ainsworth \& Bisby's dictionary of the fungi. 8. ed. Wallingford:CAB international, 1995.

HENNEN, J.F., HENNEN, M.H. \& FIGUEIREDO, M.B. Índice das ferrugens (Uredinales) do Brasil. Arquivos do Instituto Biológico. São Paulo (supl. 1):1-201. 1982.

KARL, A.C., SOUZA, R.M. \& MATTOS, J.K.A. Patogenicidade de Meloidogyne javanica em quatro espécies de plantas medicinais. Horticultura Brasileira 15:118-121. 1997.

MARTINS, E.R., CASTRO, D.M. de, CASTELLANI, D.C. \& DIAS, J.E. Plantas medicinais. Viçosa: UFV, 1995.

MATTOS, J.K.A. Plantas medicinais - problemas e possibilidades. Horticultura Brasileira 1:5-10. 1983.

MATTOS, J.K.A. \& DIANESE, J.C. Effect of light, temperature, and leaf age upon infection of Pfaffia glomerata (Spreng) Pedersen by urediniospores of Uromyces platensis Speg. Resumos, $25^{\circ}$ Congresso Brasileiro de Fitopatologia, Gramado, RS. 1992. p.162.

MATTOS, J.K.A. \& DIANESE, J.C. Studies on urediniospore germination of Uromyces platensis causal agent of rust on Pfaffia glomerata. Fitopatologia Brasileira 21:59-61. 1996.

MENDES, M.A.S., SILVA, V.L., DIANESE, J.C., FERREIRA, M.A.S.V., SANTOS, C.E.N., GOMES NETO, E., URBEN, A.F. \& CASTRO, C. Fungos em plantas no Brasil. Brasília: EMBRAPA, 1998. 
SCHUTA, L.R., LIMA, M.L.R.Z.C. \& CRACHINESKI, J.J. Ocorrência de ferrugem em plantas de erva cidreira brasileira (Lippia alba, N.E.Br.). Resumos, $30^{\circ}$ Congresso Brasileiro de Fitopatologia, Poços de Caldas, MG. 1997. p.307.

SOUZA, R.M., MATTOS, J.K.A. \& KARL, A.C. Avaliação preliminar da reação de plantas medicinais a Meloidogyne javanica e
M. incognita. Horticultura Brasileira 13:209-211. 1995.

URBEN, A.F., MATTOS, J.K.A. \& MENDES, M.A.S. Fungos associados a manchas de folhas em plantas de uso medicinal, no Distrito Federal. Fitopatologia Brasileira 12:390-394. 1987.

VIÉGAS, A.P. Alguns fungos do Brasil 4 - Uredinales. Bragantia 5:1144. 1945. 